\title{
Coral disease prevalence estimation and sampling design
}

\author{
Eric Jordán-Dahlgren ${ }^{\text {Corresp., }}{ }^{1}$ ，Adán G Jordán-Garza ${ }^{2}$ ， Rosa E Rodríguez-Martínez ${ }^{1}$ \\ 1 Instituto de Ciencias del Mar y Limnología, Universidad Nacional Autónoma de México, Puerto Morelos, Quintana Roo, México \\ ${ }^{2}$ Facultad de Ciencias Biológicas y Agropecuarias, Universidad Veracruzana, Tuxpan, Veracruz, México \\ Corresponding Author: Eric Jordán-Dahlgren \\ Email address: jordan@cmarl.unam.mx
}

In the last decades diseases have changed coral communities' structure and function in reefs worldwide. Studies conducted to evaluate the effect of diseases on corals frequently use modified adaptations of sampling designs that were developed to study ecological aspects of coral reefs. Here we evaluate how efficient these sampling protocols are by generating virtual data for a coral population parameterized with mean coral density and disease prevalence estimates from the Caribbean scleractinian Orbicella faveolata at the Mexican Caribbean. Six scenarios were tested consisting of three patterns of coral colony distribution (random, randomly clustered and randomly over-dispersed) and two disease transmission modes (random and contagious). The virtual populations were sampled with the commonly used method of belt-transects at variable sample unit sizes $(10 \times 1 \mathrm{~m}, 10 \times 2$ $\mathrm{m}, 25 \times 2 \mathrm{~m}, 50 \times 2 \mathrm{~m}$ ). Results showed that the probability of obtaining a mean coral disease prevalence estimate of $\pm 5 \%$ of the true prevalence value was low (range: $11-48 \%$ ) and that two sample comparisons achieved rather low power, unless very large effect sizes existed. Such results imply low statistical confidence to assess differences or changes in coral disease prevalence. The main problem identified was insufficient sample size because local mean colony size, density and spatial distribution of targeted coral species was not taken into consideration to properly adjust the sampling protocols. 


\section{Coral disease prevalence estimation and sampling design}

2

3 Eric Jordán-Dahlgren ${ }^{1 *}$, Adán Guillermo Jordán-Garza ${ }^{2}$, Rosa Elisa Rodríguez-Martínez ${ }^{1}$

$4{ }^{1}$ Instituto de Ciencias del Mar y Limnología, Universidad Nacional Autónoma de México,

5 Puerto Morelos, Quintana Roo, México.

$6{ }^{2}$ Facultad de Ciencias Biológicas y Agropecuarias. Universidad Veracruzana, Tuxpan, Veracruz, 7 México.

8

$9 *$ Corresponding author

11 Email address: jordan@cmarl.unam.mx 


\section{Abstract}

15 In the last decades diseases have changed coral communities' structure and function in reefs worldwide. Studies conducted to evaluate the effect of diseases on corals frequently use modified adaptations of sampling designs that were developed to study ecological aspects of coral reefs. Here we evaluate how efficient these sampling protocols are by generating virtual data for a coral population parameterized with mean coral density and disease prevalence estimates from the Caribbean scleractinian Orbicella faveolata at the Mexican Caribbean. Six scenarios were tested consisting of three patterns of coral colony distribution (random, randomly clustered and randomly over-dispersed) and two disease transmission modes (random and contagious). The virtual populations were sampled with the commonly used method of belt-transects at variable sample unit sizes $(10 \times 1 \mathrm{~m}, 10 \times 2 \mathrm{~m}, 25 \times 2 \mathrm{~m}, 50 \times 2 \mathrm{~m})$. Results showed that the probability of obtaining a mean coral disease prevalence estimate of $\pm 5 \%$ of the true prevalence value was low (range: $11-48 \%$ ) and that two sample comparisons achieved rather low power, unless very large effect sizes existed. Such results imply low statistical confidence to assess differences or changes in coral disease prevalence. The main problem identified was insufficient sample size because local mean colony size, density and spatial distribution of targeted coral species was not taken into consideration to properly adjust the sampling protocols.

\section{Keywords}

Coral, Disease prevalence, Sampling design, Statistical power 


\section{Introduction}

37 Unprecedented coral disease epizootics have changed the structure and function of coral reefs

(Aronson \& Precht, 2001; Bruno et al., 2007; Harvell et al., 2007). The causes of these epizootics are linked to environmental degradation due to global warming (Hoegh-Guldberg et al., 2007; Bruno et al., 2007; Eakin et al., 2010), and local impacts, such as eutrophication (Bruno et al., 2003; Kaczmarsky et al., 2005), pollution (Klaus et al., 2007), overfishing (Mumby et al., 2006), sedimentation (Rogers, 1990; Fabricious, 2005), dredging and coastline modification (Miller et al., 2016), plastic waste (Lamb et al., 2018) and diving activities (Lamb et al., 2014).

Our ability to fully understand the dynamics of coral diseases on coral reefs is hampered by the complex functioning of the coral holobiont and the limited knowledge of coral-disease etiologies (Ainsworth et al., 2011; Work \& Meteyer, 2014). Nevertheless, recent reports indicate that moderating or eliminating local impacts can reduce coral disease susceptibility (Fabricious, 2005; Kaczmarsky et al., 2005; Lamb et al., 2014). Therefore, sound assessments of the diseases effect are necessary to support expensive managing actions.

One of the most convenient disease assessments is to estimate prevalence (the proportion of affected individuals) and although simple in principle, prevalence estimates with acceptable accuracy and precision are not easy to obtain. Logistical constraints of underwater work make surveys on coral reefs a challenge and perhaps that is why proven and practical coral ecologicalsampling protocols are often adapted to estimate disease prevalence (Supplemental Table S1). However, by doing so, the sampling protocol is not specifically tailored to answer the question posed by a prevalence analysis which is related to a binomial condition (diseased or not). Two relevant statistically concerns arise: 1) the coral colonies sampled are not independent and 2) the 
59 necessary sample size is not properly estimated. These issues may result in low statistical power,

60 implying the possibility of 1) low probability of accurate mean estimates and of finding true

61 effects, 2) low reliability when claiming a significant effect and 3) an exaggerated estimate of the

62 magnitude of the effect when this one is true (Conner et al., 2000; Salman, 2003; Nusser et al.,

63 2008; Button et al., 2013). To evaluate the importance of these issues when estimating coral

64 disease prevalence in Caribbean key reef-building species, whose colonies tend to be large, we

65 used a simulation approach parametrized by using real data on yellow-band syndrome affecting

66 the coral Orbicella faveolata in a Mexican Caribbean reef. The sampling of virtual coral

67 populations, with known disease prevalence, allowed us to evaluate the accuracy and precision

68 of prevalence estimates obtained by commonly used sampling protocols, as well as their power

69 in comparisons for a given effect size.

71 Methods

72 To parameterize the simulations we used mean density and prevalence estimates as obtained

73 from ecological surveys adapted to include coral diseases and other signs of colony conditions.

74 We selected the data from the reef building coral Orbicella faveolata affected by the yellow-

75 band syndrome in Mahahual reef, in the Mexican Caribbean, in July 2006. The sampling

76 protocol for the site consisted of four sampling stations in the fore-reef, separated from each

77 other by at least $500 \mathrm{~m}$, in a depth range of 10-15 m. Each station comprised six $25 \times 2 \mathrm{~m}$ belt-

78 transects that were randomly deployed in an area of aproximately $100 \times 100 \mathrm{~m}$. The mean

79 prevalence of the yellow-band syndrome in 756 O. faveolata colonies surveyed in 24 belt-

80 transects $\left(1200 \mathrm{~m}^{2}\right)$ at the Mahahual reef site was $16.2 \%$. 
81 Simulated scenarios: Point pattern distributions (R spatstat, Baddeley et al., 2015) were

82

83

84

85

86

87

generated in a sampling frame area of $1500 \mathrm{~m}$ by $200 \mathrm{~m}$, by using a point density of 0.63 colonies $\mathrm{m}^{-2}$ and a proportion of "diseased" points of 0.162. Each point represented the center of a simulated coral colony, thus equivalent to real surveys where only colonies whose centers lie within the boundaries of the sample unit (i.e. belt-transect) are included (Zvuloni et al., 2008). Given that the spatial distribution of $O$. faveolata at the site is unknown, six different scenarios were simulated, based on three spatial point pattern distributions (random, randomly clustered, and randomly over-dispersed), each of them with two disease transmission modes (random and contagious). The random-disease transmission mode simulation pertains to water-borne transmission with no particular resistance on the host population and every colony had the same probability of being diseased (Jolles et al., 2002). The simulated diseased points were marked by means of a random binomial generator (package binom in R, Dorai-Raj, 2014). To simulate a possible contagious situation the probability of being diseased was dependent on the distance to a diseased neighbor, thus a nearest neighbor distance criteria determined the probability of transmission. Given the lack of better information, we subjectively assigned the probability of being diseased as a function of decreasing nearest neighbor distance percentiles $(0.5,0.7,0.8,0.9$ and 0.99 ) and used these probabilities on a random binomial generator to mark the diseased colonies on the virtual populations. In this way the closer the colonies were to each other, the higher the probability of being assigned a disease mark. Given the random processes involved on these simulations, the final coral density and disease prevalence varied around our initial target values, so to verify that the expected types of distribution pattern and disease transmission modes were achieved, we employed plots (Supplementary Figs. 1-3) of Monte Carlo confidence bands for Bezag's transformation of Ripley`s K (Baddeley et al., 2015; see supplementary 
104 material codes:1. Scenarios \& Ripley,R). Ripley`s K is a spatial analysis technique that

105 estimates the expected number of points within an increasing radius $r$ from an arbitrary point and

106 Besag's L is the normalization of $\mathrm{K}$ by way of square root transformation. With this technique

107 the point distribution patterns (random, clustering or overdispersion) can be described as a

108 function of distances by comparing deviations to a completely spatial randomness (CSR)

109 condition whose value after normalization is 0 . Thus positive values indicate clustering while

110 negative ones overdispersion (see supplementary figs)

112 Choosing a single scenario to perform simulated surveys: Given that we had no information on the spatial arrangement neither of Orbicella faveolata in general or of the diseased colonies in particular, we tested the six simulated scenarios with a similar sampling protocol as the one used in situ (i.e. 24 transects of $25 \times 2 \mathrm{~m}$ in four stations) to determine which of the six scenarios better reproduced mean density and prevalence field estimates (see supplementary material codes: 2 . Sampling \& estimates) Although our own estimates might be inaccurate, we took this approach to be able to simulate the case of a single coral host that has relatively low densities, but can reach large sizes, and is affected by a particular disease sign. Thirty independent surveys per scenario were performed to obtain colony density and prevalence estimates, each survey estimates were compared to the real field estimates. The abundance comparisons were done with negative binomial generalized linear models (R package MASS, Venables \& Ripley, 2002), that were validated if no significant residual over-dispersion was present, estimated as 1-pchisq

124 (model null deviance, model residual degrees of freedom). For disease prevalence comparisons, quasibinomial GLM's were used and considered acceptable if there was no significant over dispersion of standardized deviance residuals within 95\% confidence envelopes ( $\mathrm{R}$ package 
127 binomTools; Christensen \& Hansen, 2011; see supplementary material codes:3. Comparison of

128 scenarios to Mh data.R). The scenario showing fewer significant differences in 30 trials, was

129 selected for the performance tests of sampling protocols.

131 Testing the accuracy and precision of common survey methods: To test the theoretical

132 performance of coral disease prevalence sampling protocols, we compiled a list of methods

133 reported in the literature (Supplementary Table S1). Although sampling methodologies varied

134 among the studies that were reviewed, the majority used belt-transects, as the sampling unit, and

135 in average five belt-transects within a single sampling site. The size of the belt-transects in these

136 studies was variable, but the most common dimensions were $10 \times 1 \mathrm{~m}, 10 \times 2 \mathrm{~m}, 25 \times 2 \mathrm{~m}$ and 50x2

$137 \mathrm{~m}$. To determine the precision and accuracy of coral disease prevalence estimates obtained by a

138 protocol of five transects for each of the different belt-transect sizes, 100 independent samplings

139 were carried out for each belt-transect size protocol (see supplementary material codes: 4.

140 Accuracy of Estimates.R). Mean prevalence estimates were pooled to increase sample size and

141 then calculate the credible interval for binomial means (R package binom; Dorai-Raj 2015).

143 Testing the achieved power of two sample comparisons: For each sampling protocol, we randomly select two out of the 100 surveys to estimate the achieved power in a two-sample comparison. Power was estimated by a power sample size function for proportions (R package stats, R Core Team, 2016). By repeating this procedure 100 times, predicted power curves as a

147 function of effect size were generated as smooth splines, using generalized additive models 148 adjusted for a beta regression distribution. Model validation was done through the package's gam 
149 check (R package mgcv; Wood, 2011). Effect size was determined by means of Cliff's delta a

150 non-parametric procedure that computes ordinal variables, and thus is appropriate to deal with

151 proportions (R package epiR; Cliff, 1993; Stevenson et al., 2017 (see supplementary material

152 codes: 5. Power and effsize in two sample comparisons.R). All statistical tests and simulation

153 procedures were performed in R and RStudio. The code for the sampling simulation and

154 accuracy estimates can be found in supplementary materials.

\section{Results}

156 Choosing a single scenario to perform simulated surveys observations

157 The reef scenario with a random coral-colony distribution and a random spatial pattern of

158 diseased corals $(\mathrm{R} \& \mathrm{rt})$ yielded similar results to the filed data estimates when sampled with 24

159 belt-transects of $25 \mathrm{~m} \times 2 \mathrm{~m}$ than the data estimate (Table 1). Similar results were obtained for

160 the over-dispersed coral colony distribution and the random coral disease transmission scenario

161 (D \& rt) (Table 1). Additional comparisons between these two scenarios showed redundant

162 results and thus the $\mathrm{R} \& \mathrm{rt}$ scenario was selected to test the performance of the sampling

163 protocols commonly used in coral disease prevalence surveys.

164

165

\section{Accuracy and precision of common survey methods}

The accuracy and precision of the most common sampling protocols used in coral disease

167 prevalence surveys (Supp. Table S1) was evaluated using five belt-transects of variable size $(10 \times 1 \mathrm{~m}, 10 \times 2 \mathrm{~m}, 25 \times 2 \mathrm{~m}$ and $50 \times 2 \mathrm{~m})$. For each one of these protocols the belt-transects were randomly deployed in a "site" of 100x100 m which was randomly selected within the 1500x200 m sample frame of the R \& rt scenario (mimicking the close layout of transects within a site). 
171 For each of the four protocols, 100 independent samplings were made. The precision and

172 accuracy of the estimates varied widely within and between the different belt-transect sizes (Fig.

173 1). For the smallest sample unit design $(10 \times 1 \mathrm{~m})$, the estimated mean coral disease prevalence

174 ranged from $0.1 \%$ to $36 \%$ and had large credible confidence intervals in all cases (Fig. 1). In this

175 protocol the probability of obtaining a mean prevalence estimate of $\pm 5 \%$ of the true disease

176 prevalence value $(16.2 \%)$ was $11 \%$. In contrast, the mean disease prevalence estimated with the

177 largest sample unit $(50 \times 2 \mathrm{~m})$ ranged between $10 \%$ and $21 \%$, and had shorter credible intervals

178 (Fig. 1). However, the probability of obtaining a mean coral disease prevalence estimate of $\pm 5 \%$

179 of the true prevalence was still low (48\%).

180 While for some surveys estimating gross disease prevalence is all that is required, the low

181 accuracy and precision of the estimates imply that comparison between sites or times may not be

182 reliable unless the expected effect size was large. To assess this possibility, we estimated the

183 power for 100 independent two-sample tests for each protocol by randomly selecting pairs of

184 their corresponding 100 samplings (Fig. 2). Calculated Cliff's delta effect sizes ranged from

185 zero (no effect) to 0.8 (highest effect) and because tests are of similar context it is linearly

186 related. We found that in all cases the power was positively related to the magnitude of the effect

187 size and that, for a given effect size, the power of the test increased from the smallest to the

188 largest sample unit as sample size increased (Fig. 2). Only two out of 100 comparisons of the

189 largest sampling unit $(50 \times 2 \mathrm{~m})$ protocol reached the customary power level of 0.8 . Furthermore,

190 few tests had power values above 0.5 (less than 20\%), whereas the power level of the large

191 majority was below 0.4 (Fig. 2). The smoothed predicted lines in the same figure shows that the

192 low power trend is consistent for each belt-transect size. 


\section{Discussion}

195 Coral reef surveys are usually conducted by SCUBA diving making it difficult to efficiently

196 sample coral communities composed of multispecific assemblages living in a complex

197 tridimensional structure. This problem has promoted the use of simple methods to conduct

198 quantitative surveys of corals, such as the belt-transect and other type of quadrats as sample

199 units. These methods have frequently been modified to assess coral disease prevalence (Supp.

200 Table S1). The results of this work, however, suggest that this practice leads to poor accuracy

201 with wide credible intervals when estimating coral disease prevalence of a host that can reach

202 large sizes and thus relative low number of colonies per belt-transect area; a common case in

203 Caribbean massive reef-building corals. In this situation, the power in two sample comparisons

204 is low, unless very large effect sizes exist; thus hampering our ability to confidently assess

205 changes in disease prevalence. As the power of a test is the likelihood that an effect will be

206 detected, when indeed it exists, a high probability for a false negative (accepting the null

207 hypothesis when it's false) is of much concern when conducting coral disease assessments.

208 We found that ecological survey methods that have been repeatedly tested to be reliable to assess

209 community composition or benthic coverage (Kinzie \& Snider, 1978; Jokiel et al., 2015) may be

210 poor to estimate disease prevalence. The difference in efficiency lies in the purpose of the

211 sampling design, ecological surveys are designed to address a multivariate problem, mainly the

212 relative abundance of species or categories, which can't be reasonable sampled without the use

213 of some sort of area encompassing method such as quadrats, transects, etc., that become the

214 effective sampling unit. In contrast, disease surveys should be designed to deal with a single

215 binomial univariate variable (diseased or not) most commonly of a single kind (species, forms,

216 etc.) where the individual is the natural sampling unit, in our case a coral colony, and not the 
217 belt-transect. Therefore, unlike ecological surveys, in disease surveys the necessary sample size

218 for a given precision and accuracy is a function of the proportion of diseased colonies and not of

219 the number of belt-transect (or quadrats, circles, etc.). So, when using belt-transects they should

220 be large enough to sample a sufficient number of colonies and, for this to occur, the size and

221 shape of the belt-transects should be determined as a function of coral colony size and density of

222 the target species within the sampled area. For instance, in the Mahahual reef survey, the mean

223 transect abundance of Agaricia agaricites in our $25 \times 2 \mathrm{~m}$ belt-transects was 243 colonies, but the

224 mean abundance for the much larger Orbicella faveolata colonies was of only 34 colonies.

225 Estimation of disease prevalence in key reef building species that tend to have large colonies (i.e.

226 Acropora, Orbicella and Pseudodiploria in the Caribbean) using protocols not specifically

227 tailored for the properties of the target species, will likely result in too gross and inaccurate

228 estimates; more so as the larger the colony, the fewer will be surveyed in a belt-transect.

229 While a sufficient sample size is indispensable to obtain a coral disease prevalence estimator,

230 with a desired accuracy, the reliability of the estimate depends on obtaining representative

231 samples (Green and Green, 1979). In statistical sense representative samples can only be

232 obtained if all coral colonies of the target species, within the sampling frame, have the same

233 probability of being sampled (Casella, 2008; Lohr, 2009). However, when data are obtained by

234 belt-transects, no other colonies outside the transects area are to be sampled and so colonies

235 within the transect are not independently selected. Therefore, the magnitude of sampling bias

236 could not be assessed and there is no way to estimate to what extent collected data represents the

237 population under study, or to estimate the reliability of the prevalence values estimates (Lohr,

238 2009). Additionally, belt-transect deployment is usually done by convenience or selective criteria

239 and while recognizing that very experienced researches may make a "more" representative 
240 deployment, it still is a non-probabilistic sampling approach that further increases bias and

241 chance of pseudoreplication (Hulbert, 1984; but see Davies and Grey, 2015). To our knowledge,

242 only a few coral studies have made a probabilistic approach for coral sampling design (Smith et

243 al., 2011), and thus statistically sound to generalize their results to the reef system under study.

244 A lack of representative samples or insufficient sample size may render impossible to detect

245 valid statistical differences in coral disease prevalence between sites and through time, due to the

246 low power of the tests. Moreover, low statistical power may also result in a deflation or inflation

247 of the true effect size that result from sampling variation and random errors leading, in the case

248 of inflation, to false positives when based on thresholds of statistical significance (Button et al.,

249 2013). In this context, of hypothesis testing criteria, it should be remembered that rejection levels

250 at $\alpha=0.95$ and power $=0.80$ have no other reason than custom. A power of 0.8 means that we

251 are willing to accept a $20 \%$ chance of committing a type II error (a false negative), a margin of

252 error that may be wide for critical coral disease decisions. For instance, if the interest is in

253 detecting the effect of actions taken to minimize coral stress by reducing the residual waters

254 output to the reef environment, a false negative may imply that no actions are taken or that

255 planned actions are halted.

256 The results of this work allow us to suggest that coral disease prevalence surveys in the case of a

257 single target species with low abundance should consist of several randomly sampling units

258 deployed throughout the entire selected sample space, in order to ascertain representativeness

259 and better estimate disease variability at the study site. If comparisons are to be made using belt-

260 transects, the sample size design effect compensation due to non-random sampling should be

261 determined, as sample size formulas assume simple random sampling. These recommendations

262 will improve the quality of the estimates of coral disease prevalence, but will not allow making 
263 valid generalizations if quadrat-like sampling units are employed, unless sample-size and

264 representativeness are statistically adequate. However, by minimizing the sources of bias

265 stronger inferences could be made by issuing precise and credible statements (Qian, 2014),

266 supported by detailed description of the sampling design rationale in relation to targeted species

267 size and abundance within the selected sample space.

\section{Conclusion}

270 Ecological survey methods commonly used to assess scleractinian coral community structure

271 may be poor to estimate disease prevalence whenever large coral colony species are targeted (or

272 rare species), unless the sample units used frequently (i.e. belt-transects, quadrats) are large

273 enough. Insufficient sample size results in low power tests and statistical confidence when

274 making comparisons in time and space. Coral disease prevalence surveys should be specific for a 275 targeted species within a relatively homogeneous reef sample space in terms of its distribution 276 pattern and colony size.

278 Acknowledgments We thank M.A. Maldonado, L. Vázquez-Vera, D. Baker and J. Andras for assistance in the field. This work was supported by the Coral Disease Working Group of the

280 Global Environment Facility Coral Targeted Research program and by the Instituto de Ciencias 281 del Mar y Limnología, UNAM. Thanks to CRAN Foundation and citarlo.... 
284 Ainsworth, T.D., Wasmund, K., Ukani, L., Seneca, F., Yellowlees, D., Miller, D. \& Leggat, W. 285 2011. Defining the tipping point. A complex cellular life/death balance in corals in response to 286 stress. Scientific Reports, 1, 160.

287 Aronson, R.B. \& Precht, W.F. 2001. White-band disease and the changing face of Caribbean 288 coral reefs. Hydrobiologia, 460, 25-38.

289 Baddeley, A., Rubak, E. \& Turner, R. 2015. Spatial Point Patterns: Methodology and 290 Applications with R. London: CRC Press. 828 p.

291

292

293

294

295

296

297

298

299

300

301

302

303

304

305

306

307

308

309

310

311

312

313

314

315

316

317

318

319

320

321

Bruno, J.F., Petes, L.E., Harvell, C.D. \& Hettinger, A. 2003. Nutrient enrichment can increase the severity of coral diseases. Ecology Letters, 6(12), 1056-1061.

Bruno, J.F., Selig, E.R., Casey, K.S., Page, C.A., Willis, B.L., Harvell, C.D., Sweatman, H. \& Melendy, A.M. 2007. Thermal stress and coral cover as drivers of coral disease outbreaks. PLoS Biology, 5, 1220-1227.

Button, K.S., Ioannidis, J.P.A., Mokrysz, C., Nosek, B.A., Flint, J., Robinson E.S.J. \& Munafò, M.R. 2013. Power failure: why small sample size undermines the reliability of neuroscience. Nature Reviews | Neuroscience, 14, 365.

Casella, G. 2008. Statistical design. Springer; Berlin, Germany. 307 p.

Christensen, R.H.B. \& Hansen, M.K. 2011. binomTools: Performing diagnostics on binomial regression models. R package version 1.0-1.

Cliff, N. 1993. Dominance statistics: Ordinal analyses to answer ordinal questions. Psychological Bulletin, 114 (3), 494.

Conner, M.M., McCarty, C.W. \& Miller, M.W. 2000. Detection of bias in harvest-based estimates of chronic wasting disease prevalence in mule deer. Journal of Wildlife Diseases, 36(4), 691-699.

Davies, M.G. \& Grey, A. 2015. Don't let spurious accusations of pseudoreplication limit our ability to learn from natural experiments (and other messy kinds of ecological monitoring). Ecology and Evolution, 5(22), 5295-5304.

Dorai-Raj, S. 2014. binom: binomial confidence intervals for several parameterizations. Version 1.1-1 ed. https://CRAN.R-project.org/package=binom

Eakin, C.M., Morgan, J.A., Heron, S.F., Smith, T.B., Liu, G., Alvarez-Filip, L., ... \& Brandt, M. 2010. Caribbean corals in crisis: record thermal stress, bleaching, and mortality in 2005. PloS one, 5(11), e13969.

Fabricius, K.E. 2005. Effects of terrestrial runoff on the ecology of corals and coral reefs: review and synthesis. Marine Pollution Bulletin, 50, 125-146.

Green, R.H., \& Green, R.M. 1979. Sampling design and statistical methods for environmental biologists. John Wiley \& Sons. 257 p.

Harvell, D., Jordán-Dahlgren, E., Merkel, S., Rosenberg, E., Raymundo, L., Smith, G., ... \& Willis, B. 2007. Coral disease, environmental drivers, and the balance between coral and microbial associates. Oceanography, 20, 172-195. 
322 Hoegh-Guldberg, O., Mumby, P.J., Hooten, A.J., Steneck, R.S., Greenfield, P. Gomez, E.... \&, 323 Knowlton, N. 2007. Coral reefs under rapid climate change and ocean acidification. Science, $324318,1737-1742$.

325 Hurlbert, SH. 1984. Pseudoreplication and the design of ecological field experiments. Ecological 326 Monographs, 54(2), 187-211.

327 Jokiel, P., Rodgers, K., Brown, E., Kenyon, J., Aeby, G., Smith, W. \& Farrell, F. 2015.

328 Comparison of methods used to estimate coral cover in the Hawaiian Islands. PeerJ, 3, e954.

329

330

331

332

333

334

Jolles, A.E. Sullivan P., Alker, A.P. \& Harvell C.C. 2002. Disease transmission of Aspergillosis in sea fans: inferring process from spatial pattern. Ecology, 83(9), 2373-2378.

Kaczmarsky, L.T. Draud, M. \& Williams, E.H. 2005. Is there a relationship between proximity to sewage effluent and the prevalence of coral disease? Caribbean Journal of Science, 41(1), 124137.

Kinzie, R.A.III \& Snider, R.H. 1978. A simulation study of coral survey reef methods. Coral Reef: Research Methods. UNESCO Monographs on Oceanographic Methodology, 5, 231-250.

Klaus, J.S., Janse, I. Heikoop, J.M., Sanford, R.A. \& Fouke, B.W. 2007. Coral microbial communities, zooxanthellae and mucus along gradients of seawater depth and coastal pollution. Environmental Microbiology, 9(5), 1291-1305.

Lamb, J.B., True, J.D., Piromvaragorn, S. \& Willis, B.L. 2014. Scuba diving damage and intensity of tourist activities increases coral disease prevalence. Biological Conservation, 178, 88-96.

Lamb, J.B., Willis, B.L., Fiorenza, E.A., Couch, C.S., Howard, R. \& Rader, D.N. 2018. Plastic waste associated with disease on coral reefs. Science, 359(6374), 460-462.

Lohr, L.S. 2009. Sampling: Design and Analysis. 2nd Ed. Brooks/Cole. Cengage Learning, Inc. ISBN-10:0-495-10527-9

Miller, M.W., Karazsia, J., Groves, C.E., Griffin, S., Moore, T., Wilbert, P. \& Gregg, K. 2016. Detecting sedimentation impacts to coral reefs resulting from dredging the Port of Miami, Florida USA. PeerJ, 4, e2711.

Mumby, P.J., Dahlgren, C.P., Harborne, A.R., Kappel, C.V., Micheli, F., et al. 2006. Fishing, trophic cascades, and the process of grazing on coral reefs. Science, 311(5757), 98-101.

Nusser, S. M., Clark, W. R., Otis, D. L. \& Huang, L. 2008. Sampling considerations for disease surveillance in wildlife populations. Journal of Wildlife Management, 72(1), 52-60.

Qian, S.S. 2014. Statistics in ecology is for making a "principled" argument. Landscape Ecology, 29, 937-939.

R Core Team. 2016. R: A language and environment for statistical computing. R Foundation for Statistical Computing, Vienna, Austria. URL https://www.R-project.org/.

Rogers, C.S. 1990. Responses of coral reefs and reef organisms to sedimentation. Marine Ecology Progress Series, 62(1)185-202. 
359 Salman, M.D. 2003. Surveillance and monitoring systems for animal health programs and 360 disease surveys. Salman, M.D. (Ed.). Animal disease surveillance and survey systems. Iowa 361 State Press, Iowa, pp. 3-13.

362 Smith, S.G., Swanson, D.W., Chiappone, M., Miller, S.L. \& Ault, J.S. 2011. Probability 363 sampling of stony coral populations in the Florida Keys. Environmental Monitoring and 364 Assessment, 183(1-4), 121-138.

365 Stevenson, M. 2017. epiR: Tools for the Analysis of Epidemiological Data. R package version 366 0.9-93. https://CRAN.R-project.org/package=epiR

367 Venables, W.N. \& Ripley, B.D. 2002. Modern applied statistics with S. Chambers, S.J., Hardle, 368 E.W., Tierney, S.S. (Eds.). Springer, New York.

369 Wood, S.N. 2011. Fast stable restricted maximum likelihood and marginal likelihood estimation 370 of semiparametric generalized linear models. Journal of the Royal Statistical Society (B), 73(1), $3713-36$.

372 Work, T. \& Meteyer C. 2014. To understand coral disease, look at coral cells. EcoHealth, 11(4), 373 610-618.

374 Zvuloni, A., Artzy-Randrup, Y., Stone, L., van Woesik, R. \& Loya, Y. 2008. Ecological size375 frequency distributions: how to prevent and correct biases in spatial sampling. Limnology and 376 Oceanography: Methods, 6(3), 144-153. 
Figure 1

Mean estimated disease prevalence

Mean Estimated Prevalence (dots) and 95\% Wilson binomial confidence interval (vertical bars) of 50 randomly selected surveys of the simulated 1500 by $200 \mathrm{~m}$ reef zone. Each survey comprises one randomly deployed sample station/site $(100 \mathrm{~m}$ by $100 \mathrm{~m})$, within the reef zone. Each sampling station consisted of five randomly deployed belt-transects and the right axis text indicate the belt-transect dimensions for each panel.

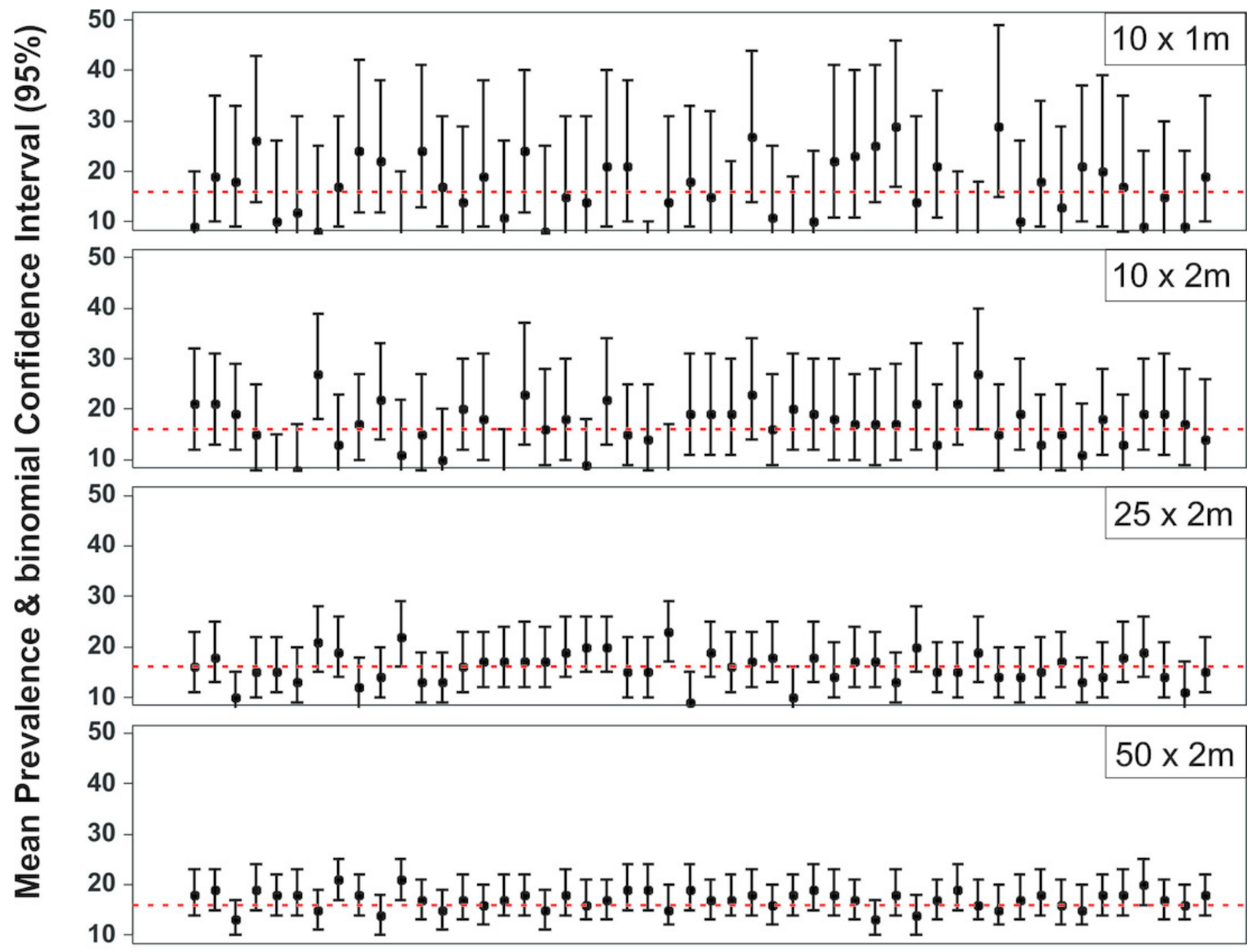

Independent surveys of the simulated reef zone 
Figure 2

Power of a two-sample comparison as function of the effect size for 100 two-sample station comparisons per sampling designs.

Open circles indicate the individual two sample power estimates. The solid line corresponds to a predict curve generated with a gam modeled with a beta regression distribution. Each sampling station consisted of five randomly deployed belt-transects and the upper text indicate the belt-transect dimensions for each curve and coresponding dat points set.

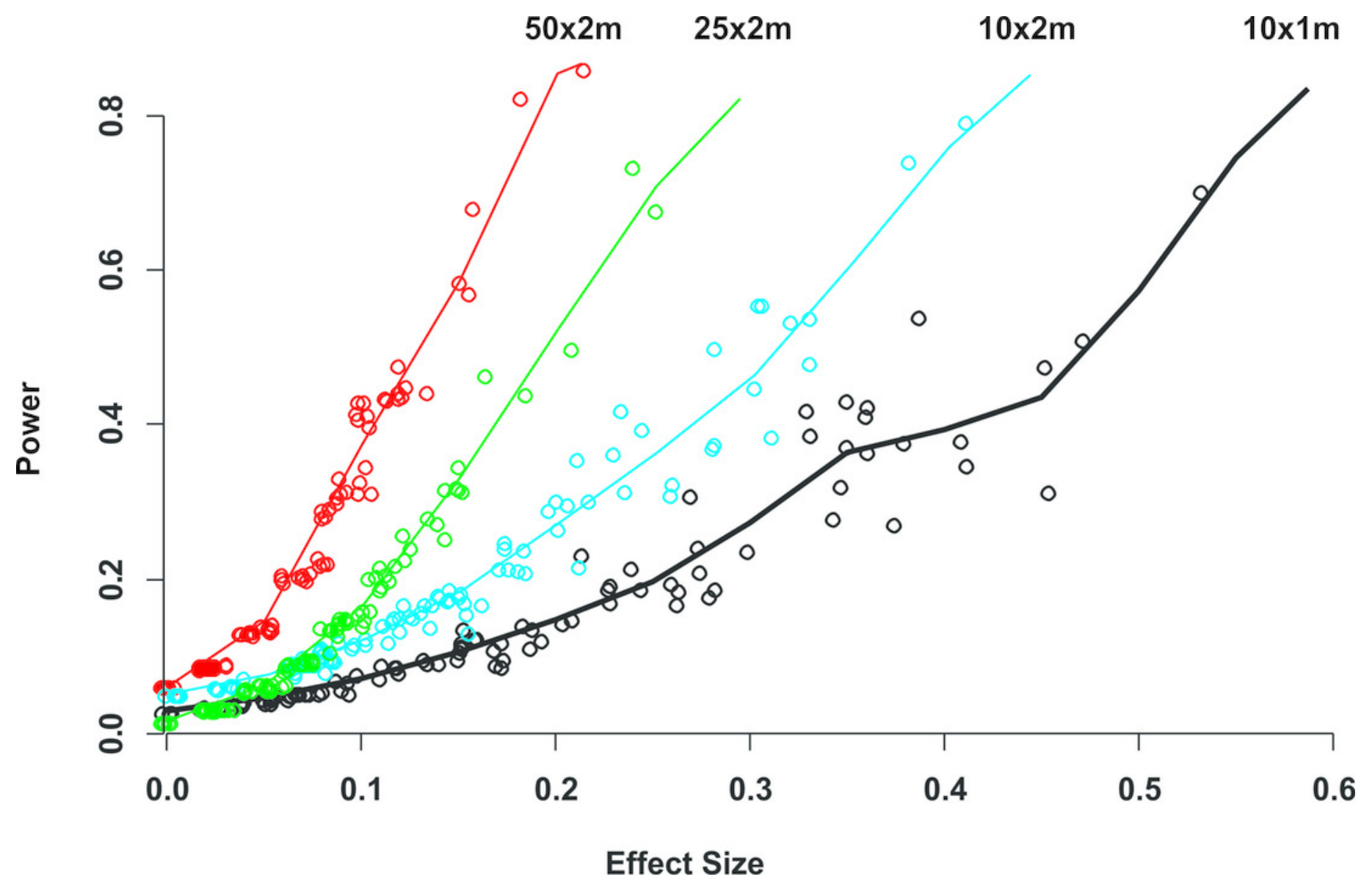




\section{Table $\mathbf{1}$ (on next page)}

Comparisons between field data and simulations of coral density and disease prevalence 
1

\begin{tabular}{|l|l|l|}
\hline Scenario & $\begin{array}{l}\text { Coral (point) } \\
\text { mean density }\end{array}$ & $\begin{array}{l}\text { Coral (point) } \\
\text { mean disease prevalence }\end{array}$ \\
\hline R \& rt & 0.0 & 0.0 \\
\hline R \& ct & 26.7 & 0.0 \\
\hline C \& rt & 20.0 & 0.0 \\
\hline C \& ct & 36.7 & 16.7 \\
\hline D \& rt & 0.03 & 0.0 \\
\hline D \& ct & 16.7 & 0.0 \\
\hline
\end{tabular}

2 